Published in final edited form as:

Curr Hematol Malig Rep. 2012 March ; 7(1): 13-20. doi:10.1007/s11899-011-0106-x.

\title{
Immune Reconstitution in Chronic Lymphocytic Leukemia
}

\author{
John C. Riches, \\ Barts Cancer Institute, Queen Mary University of London, 3rd Floor John Vane Science Centre, \\ Charterhouse Square, London EC1M 6BQ, UK
}

Alan G. Ramsay, and

Barts Cancer Institute, Queen Mary University of London, 3rd Floor John Vane Science Centre, Charterhouse Square, London EC1M 6BQ, UK

\section{John G. Gribben}

Barts Cancer Institute, Queen Mary University of London, 3rd Floor John Vane Science Centre, Charterhouse Square, London EC1M 6BQ, UK

John C. Riches: johnriches@doctors.org.uk; Alan G. Ramsay: a.ramsay@qmul.ac.uk; John G. Gribben: j.gribben@qmul.ac.uk

\section{Abstract}

Chronic lymphocytic leukemia (CLL) is associated with a profound immune defect, which results in increased susceptibility to recurrent infections as well as a failure to mount effective antitumor immune responses. Current chemotherapy-based regimens are not curative and often worsen this immune suppression, so their usefulness is limited, particularly in the frail and elderly. This article reviews the immune defect in CLL and discusses strategies aimed at repairing or circumventing this defect. In particular, it focuses on recent developments in the areas of CD40 ligand (CD40L/ CD154) gene therapy, immunomodulatory agents such as lenalidomide, and adoptive transfer of T cells bearing chimeric antigen receptors.

\section{Keywords}

Chronic lymphocytic leukemia; CLL; Immune suppression; Immune reconstitution; CD40 ligand; Gene therapy; Lenalidomide; Chimeric antigen receptor; Adoptive T-cell therapies

\section{Introduction}

B-cell chronic lymphocytic leukemia (CLL) is a disease caused by a clonal expansion of small, mature B lymphocytes. Although it is often detected as a consequence of a lymphocytosis in otherwise asymptomatic patients, patients with more advanced disease can exhibit a variety of symptoms including weight loss, sweats, lymphadenopathy, splenomegaly, and bone marrow failure. A major feature of CLL is that patients are susceptible to recurrent infections, which are a major cause of morbidity and mortality in

Correspondence to: John G. Gribben, j . gribben@qmul . ac . uk.

Disclosure Conflicts of Interest: J. Riches: none; A. Ramsay: none; J. Gribben: Consultancy fees from Merck, Celgene; honoraria from Roche/Genentech, GlaxoSmithKline, Mundipharma. 
this disease. Chemotherapy remains a key component of current therapies, but it is often potently immunosuppressive, exacerbating the immune defect and patients' susceptibility to infections. Combination chemoimmunotherapy with fludarabine, cyclophosphamide, and rituximab is the current "standard of care" but appears too toxic for the elderly and those with comorbidities, and it is not curative $[1 \bullet, 2 \bullet \bullet]$. Furthermore, the response to chemoimmunotherapy is still unacceptably poor in well-characterized high-risk subsets of patients.

Attempting to reconstitute the immune response in CLL is attractive for three reasons. First, the immune surveillance hypothesis suggests that in order to produce clinically detectable disease, the malignant CLL B cells must have evolved strategies of evading or suppressing the immune system, especially the anticancer effects of $T$ cells [3]. Therefore, successful immune reconstitution should lead to repair of antitumor immunity and durable clinical responses. Second, T cells provide "help" to B cells as part of a healthy immune system, by stimulating the B cells to proliferate, inducing B-cell antibody class switching, and promoting plasma cell differentiation. In CLL, there is evidence that $\mathrm{T}$ cells have been skewed to provide "help" for the malignant B cells, and therefore successful immune reconstitution should reduce the availability of T-cell-derived pro-CLL factors, leading to "starvation" of the CLL cells. Finally, even in the absence of any antitumor effects, immune reconstitution would benefit patients by enabling them to fight infections more effectively and would counteract the immune suppression induced by both the disease and current therapies. Strategies employing this approach should result in therapies that are more tolerable to vulnerable patients and show enhanced efficacy in the more challenging poorrisk subgroups.

\section{Immune Deficiency in CLL}

The immune deficiency seen in CLL is wide-ranging, resulting in increased susceptibility to bacterial, viral, and fungal infections and failure to mount an effective antitumor immune response. Nevertheless, one of the earliest observations of the immune system in this disease was that there was a paradoxical increase in the number of circulating $T$ cells, which was primarily accounted for by an increased number of CD8+ T cells, resulting in a fall in the CD4:CD8 ratio [4-6]. These $T$ cells show a variety of phenotypic and functional abnormalities. Phenotypically, they show increased expression of CD57, CD69, and HLADR, along with decreased expression of CD28 and CD62L, which would suggest activation and a shift towards a terminally differentiated effector-memory subtype [7-9]. CLL patients show oligoclonal expansions of both CD4+ and CD8+ T cells, particularly within the CD57+ subset [10-13]. It was subsequently demonstrated that these cells show specificity for cytomegalovirus (CMV), and that these CMV-specific $\mathrm{T}$ cells dominate the T-cell repertoire in seropositive patients $[14,15]$. The reason for this change is unclear, but this expansion may restrict the overall T-cell repertoire by "crowding out" $\mathrm{T}$ cells with other specificities [16].

Functionally, both CD4+ and CD8+ T cells from patients with CLL have been demonstrated to secrete increased amounts of the prototypical Th2 cytokine interleukin-4 (IL-4) [17]. Under normal circumstances, this cytokine promotes a humoral immune response, and it has 
been shown to protect CLL B cells from apoptosis by upregulating expression of the antiapoptotic molecule Bcl-2 [18-20]. Furthermore, IL-4-producing CD8+ T cells from CLL patients show increased expression of CD30 [21]. Ligation of CD30L on the surface of the CLL B cells has been shown to stimulate their production of TNF-a causing them to proliferate. Conversely, binding to $\mathrm{CD} 30 \mathrm{~L}$ on the surface of the nonmalignant B cells impairs isotype class switching and increases their sensitivity to FasL-mediated cell death [22]. In combination with the defects in T-helper cell function, this change may underlie the hypogammaglobulinemia seen in CLL [23, 24]. Expansion of CD4 + CD25+ regulatory $\mathrm{T}$ cells $\left(\mathrm{T}_{\text {regs }}\right)$ also may contribute to the immune defect in CLL. Absolute numbers of $\mathrm{T}_{\text {regs }}$ are increased in CLL, with the largest increases found in patients with the most clinically advanced disease [25-27]. Higher frequencies of $\mathrm{T}_{\text {regs }}$ have also been shown to correlate with decreased T-cell responses against viral and tumor antigens [26]. $\mathrm{T}_{\text {regs }}$ also may decrease cellular immunity by soluble IL-2 receptor secretion, inhibiting Th1 differentiation [28]. The expansion of $\mathrm{T}_{\text {regs }}$ in CLL may be due to a combination of increased formation, facilitated by intranodal CD27-CD70 interaction, and decreased sensitivity to apoptosis as a consequence of higher expression of Bcl-2 [29, 30].

Further information about the nature of the T-cell defect in CLL was provided by our work using global gene expression profiling to demonstrate that $\mathrm{T}$ cells from patients with CLL show a number of differentially expressed genes when compared with age-matched healthy donor T cells [31]. Although the T cells were not part of the tumor clone, analysis revealed altered expression of genes involved in cell differentiation and cytoskeletal formation in patient CD4+ T cells, and cytoskeletal organization, vesicle trafficking, and cytotoxicity pathways in patient CD8+ T cells. Similar alterations in cytoskeletal formation pathways could be induced in healthy allogeneic $\mathrm{T}$ cells by co-culturing them with CLL cells, in a contact-dependent manner. We have subsequently demonstrated that these changes in the expression of cytoskeletal genes translate into a functional defect in actin polymerization. The result is that $\mathrm{T}$ cells from CLL patients exhibit defective immunologic synapse formation with antigen presenting cells (APCs), a finding that could similarly be induced in healthy $\mathrm{T}$ cells by coculturing them with CLL cells [32]. Support for these findings was provided by our observations that the development of leukemia in E $\mu$-TCL1 transgenic mice induces remarkably comparable changes in gene and protein expression and T-cell function to those seen in human patients with CLL [33]. This work demonstrated that it is the CLL cells that drive the changes in $\mathrm{T}$ cells, as infusion of malignant $\mathrm{T}$ cells into healthy animals induced the gene expression and functional defects. Further studies have shown that this model also accurately mimics the shift towards an antigen-experienced phenotype observed in the human disease [34].

\section{CD40 Ligand Gene Therapy}

A further important finding in the immunobiology of CLL was the observation that patient $\mathrm{T}$ cells show reduced expression of CD40L [35]. CD40/CD40L interactions are crucial for the maturation, expansion, and survival of normal B cells. Under normal circumstances, ligation of the T-cell receptor (TCR) results in transient expression of CD40L, a class II membrane glycoprotein [36]. This can bind CD40 expressed by B cells, resulting in upregulation of adhesion molecules including intercellular adhesion molecule-1 (ICAM-1/CD54), 
lymphocyte function associated antigen- 3 (LFA-3/CD58), and co-stimulatory molecules such as CD80 and CD86 [37]. Despite expressing near-normal levels of MHC class II molecules, CLL B cells are poor antigen presenters because of reduced expression of CD80 and CD86 [38]. Pre-activation of these cells by CD40 ligation can upregulate these costimulatory molecules and significantly improve the antigen-presenting function of the CLL B cells. When CLL cells were co-cultured with mouse fibroblasts expressing human CD40L, they rapidly upregulated CD80 and CD86 and were able to prime allogeneic CD8+ T cells to show cytotoxicity towards unstimulated CLL B cells [39].

Therefore, a number of strategies have been developed to capitalize on the activating effect of CD40L on B cells. One such technique was to use adenoviral vectors to transduce CLL cells to express CD40L. In addition to inducing expression of co-stimulatory and adhesion molecules on the transduced cell, this technique can enable them to "transactivate" noninfected bystander CLL B cells. Preclinical studies demonstrated that these modified B cells were highly effective stimulators in mixed lymphocyte reactions and were able to induce generation of cytotoxic CD8+ T cells that were specific for autologous unmodified cells [40]. A subsequent clinical trial examined the safety and efficacy of infusions of autologous tumor cells that had been transduced ex vivo with murine CD40L, which was more efficiently expressed than human CD40L. This treatment was well tolerated and resulted in peripheral blood and lymph node responses, but some of the patients developed antibodies against the murine CD40L [41]. In light of this finding, a recombinant humanized CD40 binding protein, ISF35, was developed. A recent phase 1 study investigated the effect of autologous tumor cells transduced ex vivo with ISF35, in patients with CLL. The infusions were again well tolerated and were consistently followed by reductions in circulating lymphocyte counts and lymphadenopathy. After infusion, the circulating CLL B cells had increased expression of pro-apoptotic molecules CD95, DR5, p73, and BCL-2 interacting domain (Bid), which enhanced their susceptibility to apoptosis. A reduction in levels of an anti-apoptotic molecule, Mcl-1, was also noted, and these findings were also observed in patients with deletion of chromosome $17 \mathrm{p}$ [42•]. Further in vitro data have provided a rationale for combining this approach with rituximab, as stimulation of CLL B cells by CD40L sensitizes them to rituximab-induced cell death [43]. However, it has also recently been demonstrated that CLL B cells show heterogenous responses to CD40L stimulation. Patients with CLL B cells that were relatively unresponsive to CD40L showed a poor clinical outcome with a shorter time to progression, which presumably reflected less dependency on the microenvironment and higher autonomous proliferative and survival potential [44]. As a consequence, this subset of patients may well exhibit a poor response to CD40L gene therapy.

Lenalidomide Lenalidomide has been demonstrated to have significant clinical activity in CLL. In previously untreated patients, the overall response rate with single-agent lenalidomide was $56 \%$. This is comparable to the response rates of commonly used agents such as fludarabine, alemtuzumab, bendamustine, and chlorambucil when used as first-line single agents [45••]. Furthermore, a phase 2 trial has demonstrated similar activity in elderly patients ( $\ 65$ years), with lenalidomide being generally well tolerated [46•]. In light of these findings, trials of combinations of lenalidomide with more established agents are under way. 
One of the key features of lenalidomide's clinical activity in CLL is that patients generally can tolerate only lower doses $(10 \mathrm{mg})$ than are used in other hematologic malignancies such as myeloma $(25 \mathrm{mg})$. A major contributing factor to this difference is the presence of a "tumor flare" reaction, which appears to be unique to CLL. This reaction is manifested by acute swelling of involved lymph nodes, associated inflammation of the overlying skin, hepatosplenomegaly, rash, and fever. Its severity has been found to correlate with increased expression of CD40, CD80, and CD86, and consequently it has been suggested that this "flare" occurs as a result of the improvement in CLL B-cell antigen presentation, inducing an immune antitumor response [47]. The presence of a tumor flare reaction appears to correlate with clinical outcome, suggesting that mechanisms underlying this phenomenon may also account for the antitumor effect of this agent [48]. However, the existence of this phenomenon is proving a challenge to combination regimens using lenalidomide: a recent report concluded that the concurrent administration of fludarabine, rituximab, and lenalidomide was not tolerable, owing to tumor flare, idiosyncratic drug reactions, and myelosuppression [49]. The optimal use of lenalidomide in CLL remains to be determined, and rational combination therapies may seek to avoid the use of lenalidomide with T-cell depleting agents such as fludarabine, instead focusing on its use with monoclonal antibodies such as rituximab, or as a maintenance therapy.

Despite its activity in a variety of hematologic diseases, the mechanism of action of lenalidomide is not well understood. Early work identified lenalidomide's ability to inhibit production of TNF-a, IL-1 $\beta$, IL-6, and IL-12 from lipopolysaccharide-stimulated peripheral blood mononuclear cells (PBMCs) in vitro [50]. Subsequent research demonstrated that lenalidomide had co-stimulatory effects, causing T-cell activation with increased production of IL-2 and interferon- $\gamma$ and triggering tyrosine phosphorylation of CD28 with downstream activation of NF- $\kappa \mathrm{B}$ [50-52]. We have shown that the functional defect of T cells in CLL is associated with impaired actin polymerization resulting in defective immunologic synapse formation. We subsequently demonstrated that treatment of both autologous $\mathrm{T}$ cells and CLL cells with lenalidomide resulted in repair of this defect, suggesting that this repair may be a key component of this agent's activity in CLL [32]. Other investigators have shown that pomalidomide, an analogue of lenalidomide, can activate the cytoskeletal regulators Rac1 and RhoA. There is also evidence to suggest that lenalidomide has effects on the malignant B cells, inducing upregulation of CD40L, Bid, DR5, and p73, which sensitizes them to TNFrelated apoptosis-inducing ligand (TRAIL)-mediated apoptosis [53]. Lenalidomide also has effects on other nonmalignant lymphocyte subsets, having been shown to decrease the number of $\mathrm{T}_{\text {regs }}$ and to augment natural killer cell-mediated cytotoxicity [54-56].

\section{Chimeric Antigen Receptors}

A particularly interesting area of investigation, which aims to circumvent many of the problems associated with the approaches discussed above, is the adoptive transfer of $\mathrm{T}$ cells with specificity for tumor antigens (Fig. 1). The existence of a graft versus leukemia (GVL) effect and the fact that allogeneic hematopoietic stem cell transplantation remains the only curative therapy for CLL have led to this being the "holy grail" of cancer immunotherapy for many years [57]. There are two main strategies for generating tumor-specific T cells. The first involves the gene transfer of T-cell receptors (TCRs) with known specificity into 
autologous or allogeneic $\mathrm{T}$ cells, which are then expanded in vitro and infused into patients. This approach has had some successes, most notably in melanoma and in the use of T cells specific to Epstein-Barr virus to treat posttransplant lymphoproliferative disorders [58-60]. However, the recognition of the tumor antigens is MHC-restricted, so the use of these T cells must be individualized on a patient-by-patient basis according to their MHC type. Furthermore, there is a risk that a subunit of the transgenic TCR could mis-associate with a subunit of the endogenous TCR, changing the specificity of the T cell and potentially leading to autoimmunity.

The second strategy involves the use of an antibody-derived antigen-binding moiety (usually a single-chain variable fragment) fused with an internal signalling domain such as $\mathrm{CD} 3 \zeta$ to form a chimeric antigen receptor (CAR) [61]. This approach eliminates MHC restriction, enabling the same CAR to be used for several different patients. Furthermore, the use of an antibody receptor means that potential targets can be increased to include a wide range of surface proteins, sugars, and lipids [62]. The target of these CARs must be carefully selected to avoid "on-target, off-organ" effects, which potentially can occur when the antigen is also expressed on nonmalignant tissues. In the context of CLL, particularly attractive targets are CD19, CD20, CD23, and receptor tyrosine kinase-like orphan receptor 1 (ROR1). CLL B cells express high levels of CD19, in contrast to the relatively reduced expression of CD20. A disadvantage of targeting these molecules is that they are also expressed by normal B cells, so CAR T cells targeting them will also eliminate normal B cells, causing persistently impaired humoral immunity and exacerbating the immunodeficiency already present in CLL [63]. Anti-ROR1 CAR CD8+ T cells that recognize autologous CLL B cells have been successfully generated from patients with CLL. ROR1 has the advantage of being selectively expressed by malignant B cells, although it is also expressed by undifferentiated embryonic stem cells and (at low levels) in adipose tissue [64]. Similarly, anti-CD23 CAR T cells generated from CLL patients have shown cytotoxicity against autologous and allogeneic CLL cells and also have shown an in vivo antitumor effect in a xenograft murine model [65].

A number of phase $1 / 2$ clinical trials are under way using anti-CD19 CAR T cells for the treatment of B-cell malignancies [63]. Preclinical studies demonstrated that anti- CD19 CAR T cells could efficiently lyse a wide panel of human CD19+ tumor cell lines and primary malignant $\mathrm{B}$ cells and also showed antilymphoma effects in a murine model [66, 67]. The addition of a co-stimulatory domain such as CD28 has been shown to significantly improve the efficacy of CAR T cells, overcoming the reduced expression of CD80 and CD86 seen in B-cell malignancies such as CLL $[38,68]$. A clinical trial with anti-CD19 CAR T cells in a patient with advanced follicular lymphoma resulted in regression of lymphadenopathy, associated with Blymphopenia and hypogammaglobulinemia. Unfortunately, the CAR T cells did not persist long-term: the anti-CD19 CAR became undetectable at 27 weeks, and progressive disease developed at 32 weeks [69]. This group has treated two patients with CLL, with the first patient achieving a complete remission after infusions of the anti-CD19 CAR T cells. All of these patients received conditioning with fludarabine, cyclophosphamide, and high-dose interleukin-2 (IL-2) [70]. A second group has treated eight patients with relapsed purine analogue-refractory CLL in two cohorts. The first 
cohort of three patients was treated without cyclophosphamide conditioning and showed no objective responses. The next patient received lymphodepleting chemotherapy with cyclophosphamide as part of the trial design. Unfortunately, this patient rapidly developed hypotension, respiratory distress, and renal failure, and died within $48 \mathrm{~h}$ of infusion of the T cells [71]. The death was attributed to sepsis rather than to the CAR T cells directly, but it still highlights the risks associated with this therapy. A further four patients have been treated with cyclophosphamide conditioning with a reduced dose of $\mathrm{T}$ cells, and three of these patients showed disease stabilization or lymph node responses. This group showed some persistence of the anti-CD19 CAR T cells, which were detectable by immunohistochemistry in bone marrow up to 2 months after infusion [72]. This trial has highlighted the importance of the conditioning regimen in promoting T-cell engraftment and activation and sensitizing the tumor cells to cell-mediated cytotoxicity. In particular, it may be vital to eliminate $\mathrm{T}_{\text {regs }}$, which are known to be expanded in CLL and can be suppressed with fludarabine treatment $[25,26,29]$. It may also be important to eliminate other subpopulations such as immature dendritic cells and cell populations that compete for the same survival and stimulatory cytokines [63].

A further recent report documented a case of a heavily pretreated patient with refractory CLL who entered a complete remission after the adoptive transfer of second-generation antiCD19 CAR T cells. What was particularly interesting about this case was that these cells were still detectable at 6 months after the infusion and had started to express the adhesion molecule CCR7 and the interleukin-7 receptor (CD127). Both of these molecules are associated with the acquisition of a "central memory" phenotype, which is known to be important in maintaining robust and persistent antitumor immune responses. This patient received conditioning with pentostatin and cyclophosphamide and received a relatively low dose of $1.46 \times 10^{5}$ transduced $\mathrm{T}$ cells $/ \mathrm{kg}$ with no additional cytokines. There were no acute toxic effects, although the patient subsequently developed tumor lysis syndrome at day 22 , which was temporally associated with high levels of inflammatory cytokines and 1000-fold proliferation of the anti-CD19 CAR T cells [73•]. The same group also reported two further cases, one of whom also achieved complete remission after heavy pretreatment. The second patient, who also had deletion of p53, showed only a partial response to anti-CD19 CAR T cells, but required corticosteroids for persistent fevers and constitutional and cardiac symptoms at day 18 [74]. The key to the success of these CAR T cells appears to be the use of the CD137 (4-1BB) co-stimulatory domain. This domain was chosen over CD28 because it is less likely to trigger IL-2 and TNF-a secretion and thus less likely to induce a "cytokine storm" and differentiation of $\mathrm{T}_{\text {regs. }}$. In a murine model of primary human pre-B-cell acute lymphoblastic leukemia, human T cells expressing anti-CD19 CARs containing CD137 were significantly more effective than cells expressing CARs containing the CD28 signaling domain, and they showed prolonged survival [75]. Indeed, no rises were seen in these cytokines, in contrast to the increase in serum TNF- $a$ and tumor flare reaction seen with lenalidomide and the increased secretion of TNF-a by anti-CD23 CAR T cells when the CD28 domain was used $[65,76]$. The T cells still express CD28, so it is possible that these cells actually behave more like third-generation CARs, which include a combination of costimulatory domains [77]. 
Although this is an encouraging development, much further work is needed. Correlative multiparameter flow cytometry showed high expression of CD45RA, PD-1, and CD57 on both CD4+ and CD8+ CAR T cells 169 days after infusion [74]. These findings may well reflect the extensive replicative history of these cells, but they also could indicate T-cell exhaustion and incipient loss of function [78]. It is possible that this exhaustion could lead to treatment failure in the longer term, requiring further infusions of these $\mathrm{T}$ cells to maintain clinical responses. However, the converse may apply, with high expression of inhibitory receptors such as PD-1 actually acting as a brake on these cells, reducing the risk of systemic immune activation and adverse effects. Furthermore, the risk associated with profound long-term B-cell lymphopenia and hypogammaglobulinemia is still unknown; the immune deficiency that is already a feature of CLL could be exacerbated. Nevertheless, this remains an exciting area of research because of the potential of targeting of alternative tumor antigens, other co-stimulatory approaches, and the engineering of other cell types with CARs, such as natural killer (NK) cells and NKT cells [79].

\section{Conclusions}

The recent successes of CD40L gene therapy, CAR T cells, and lenalidomide have highlighted the "coming of age" of immunotherapy for CLL. These approaches offer the opportunity to provide patients with therapies that reduce rather than exacerbate the immune suppression associated with this disease, allowing treatment strategies that are viable for all patients, including the elderly and those with comorbidities. Furthermore, a particularly exciting aspect of these treatments is that they appear to be effective in subgroups of patients who have posed a particular challenge to standard therapy.

\section{References}

Papers of particular interest, published recently, have been highlighted as:

- Of importance

•• Of major importance

1. Eichhorst BF, Busch R, Stilgenbauer S, et al. First-line therapy with fludarabine compared with chlorambucil does not result in a major benefit for elderly patients with advanced chronic lymphocytic leukemia. Blood. 2009; 114:3382-3391. [PubMed: 19605849] This important clinical trial highlights the challenges of successfully treating elderly patients with CLL, emphasizing the need for novel noncytotoxic therapies for this disease.

2. Hallek M, Fischer K, Fingerle-Rowson G, et al. Addition of rituximab to fludarabine and cyclophosphamide in patients with chronic lymphocytic leukaemia: a randomised, open-label, phase 3 trial. Lancet. 2010; 376:1164-1174. [PubMed: 20888994] This phase 3 trial demonstrates the survival advantage of the addition of rituximab to fludarabine and cyclophosphamide, and underpins the current standard of care in CLL.

3. Dunn GP, Bruce AT, Ikeda H, et al. Cancer immunoediting: from immunosurveillance to tumor escape. Nat Immunol. 2002; 3:991-998. [PubMed: 12407406]

4. Catovsky D, Miliani E, Okos A, et al. Clinical significance of T-cells in chronic lymphocytic leukaemia. Lancet. 1974; 2:751-752. [PubMed: 4143015]

5. Platsoucas CD, Galinski M, Kempin S, et al. Abnormal T lymphocyte subpopulations in patients with B cell chronic lymphocytic leukemia: an analysis by monoclonal antibodies. J Immunol. 1982; 129:2305-2312. [PubMed: 6214590] 
6. Herrmann F, Lochner A, Philippen H, et al. Imbalance of T cell subpopulations in patients with chronic lymphocytic leukaemia of the B cell type. Clin Exp Immunol. 1982; 49:157-162. [PubMed: 6982129]

7. Van den Hove LE, Vandenberghe P, Van Gool SW, et al. Peripheral blood lymphocyte subset shifts in patients with untreated hematological tumors: evidence for systemic activation of the $\mathrm{T}$ cell compartment. Leuk Res. 1998; 22:175-184. [PubMed: 9593474]

8. Velardi A, Prchal JT, Prasthofer EF, et al. Expression of NK-lineage markers on peripheral blood lymphocytes with T-helper (Leu3+/T4+) phenotype in B cell chronic lymphocytic leukemia. Blood. 1985; 65:149-155. [PubMed: 3155487]

9. Rossi E, Matutes E, Morilla R, et al. Zeta chain and CD28 are poorly expressed on T lymphocytes from chronic lymphocytic leukemia. Leukemia. 1996; 10:494-497. [PubMed: 8642868]

10. Goolsby CL, Kuchnio M, Finn WG, et al. Expansions of clonal and oligoclonal T cells in B-cell chronic lymphocytic leukemia are primarily restricted to the CD3(+)CD8(+) T-cell population. Cytometry. 2000; 42:188-195. [PubMed: 10861692]

11. Rezvany MR, Jeddi-Tehrani M, Osterborg A, et al. Oligoclonal TCRBV gene usage in B-cell chronic lymphocytic leukemia: major perturbations are preferentially seen within the CD4 T-cell subset. Blood. 1999; 94:1063-1069. [PubMed: 10419899]

12. Serrano D, Monteiro J, Allen SL, et al. Clonal expansion within the CD4+CD57+ and CD8+CD57+ T cell subsets in chronic lymphocytic leukemia. J Immunol. 1997; 158:1482-1489. [PubMed: 9013995]

13. Farace F, Orlanducci F, Dietrich PY, et al. T cell repertoire in patients with B chronic lymphocytic leukemia. Evidence for multiple in vivo T cell clonal expansions. J Immunol. 1994; 153:42814290. [PubMed: 7930628]

14. Mackus WJ, Frakking FN, Grummels A, et al. Expansion of CMV-specific CD8+CD45RA+CD27T cells in B-cell chronic lymphocytic leukemia. Blood. 2003; 102:1057-1063. [PubMed: 12689926]

15. Pourgheysari B, Bruton R, Parry H, et al. The number of cytomegalovirus-specific CD4+ T cells is markedly expanded in patients with B-cell chronic lymphocytic leukemia and determines the total CD4+ T-cell repertoire. Blood. 2010; 116:2968-2974. [PubMed: 20562332]

16. Akbar AN. The silent war against CMV in CLL. Blood. 2011; 116:2869-2870. [PubMed: 20966175]

17. Mu X, Kay NE, Gosland MP, et al. Analysis of blood T-cell cytokine expression in B-chronic lymphocytic leukaemia: evidence for increased levels of cytoplasmic IL-4 in resting and activated CD8 T cells. Br J Haematol. 1997; 96:733-735. [PubMed: 9074413]

18. Dancescu M, Rubio-Trujillo M, Biron G, et al. Interleukin 4 protects chronic lymphocytic leukemic B cells from death by apoptosis and upregulates Bcl-2 expression. J Exp Med. 1992; 176:1319-1326. [PubMed: 1402678]

19. Panayiotidis P, Ganeshaguru K, Jabbar SA, et al. Interleukin-4 inhibits apoptotic cell death and loss of the bcl-2 protein in B-chronic lymphocytic leukaemia cells in vitro. Br J Haematol. 1993; 85:439-445. [PubMed: 8136263]

20. Kay NE, Han L, Bone N, et al. Interleukin 4 content in chronic lymphocytic leukaemia (CLL) B cells and blood CD8+ T cells from B-CLL patients: impact on clonal B-cell apoptosis. Br J Haematol. 2001; 112:760-767. [PubMed: 11260081]

21. de Totero D, Reato G, Mauro F, et al. IL4 production and increased CD30 expression by a unique CD8+ T-cell subset in B-cell chronic lymphocytic leukaemia. Br J Haematol. 1999; 104:589-599. [PubMed: 10086799]

22. Cerutti A, Kim EC, Shah S, et al. Dysregulation of CD30+ T cells by leukemia impairs isotype switching in normal B cells. Nat Immunol. 2001; 2:150-156. [PubMed: 11175813]

23. Foa R, Catovsky D, Brozovic M, et al. Clinical staging and immunological findings in chronic lymphocytic leukemia. Cancer. 1979; 44:483-487. [PubMed: 314324]

24. Lauria F, Foa R, Mantovani V, et al. T-cell functional abnormality in B-chronic lymphocytic leukaemia: evidence of a defect of the T-helper subset. Br J Haematol. 1983; 54:277-283. [PubMed: 6221752] 
25. Beyer M, Kochanek M, Darabi K, et al. Reduced frequencies and suppressive function of CD4+CD25hi regulatory $\mathrm{T}$ cells in patients with chronic lymphocytic leukemia after therapy with fludarabine. Blood. 2005; 106:2018-2025. [PubMed: 15914560]

26. Giannopoulos K, Schmitt M, Kowal M, et al. Characterization of regulatory T cells in patients with B-cell chronic lymphocytic leukemia. Oncol Rep. 2008; 20:677-682. [PubMed: 18695923]

27. D'Arena G, Laurenti L, Minervini MM, et al. Regulatory T-cell number is increased in chronic lymphocytic leukemia patients and correlates with progressive disease. Leuk Res. 2011; 35:363368. [PubMed: 20880586]

28. Lindqvist CA, Christiansson LH, Simonsson B, et al. T regulatory cells control T-cell proliferation partly by the release of soluble CD25 in patients with B-cell malignancies. Immunology. 2010; 133:371-376. [PubMed: 20518821]

29. Jak M, Mous R, Remmerswaal EB, et al. Enhanced formation and survival of CD4+ CD25hi Foxp3+ T-cells in chronic lymphocytic leukemia. Leuk Lymphoma. 2009; 50:788-801. [PubMed: 19452318]

30. Pallasch CP, Ulbrich S, Brinker R, et al. Disruption of T cell suppression in chronic lymphocytic leukemia by CD200 blockade. Leuk Res. 2009; 33:460-464. [PubMed: 18838168]

31. Gorgun G, Holderried TA, Zahrieh D, et al. Chronic lymphocytic leukemia cells induce changes in gene expression of CD4 and CD8 T cells. J Clin Invest. 2005; 115:1797-1805. [PubMed: 15965501]

32. Ramsay AG, Johnson AJ, Lee AM, et al. Chronic lymphocytic leukemia T cells show impaired immunological synapse formation that can be reversed with an immunomodulating drug. J Clin Invest. 2008; 118:2427-2437. [PubMed: 18551193]

33. Gorgun G, Ramsay AG, Holderried TA, et al. E(mu)-TCL1 mice represent a model for immunotherapeutic reversal of chronic lymphocytic leukemia-induced T-cell dysfunction. Proc Natl Acad Sci U S A. 2009; 106:6250-6255. [PubMed: 19332800]

34. Hofbauer JP, Heyder C, Denk U, et al. Development of CLL in the TCL1 transgenic mouse model is associated with severe skewing of the T-cell compartment homologous to human CLL. Leukemia. 2011; 25:1452-1458. [PubMed: 21606964]

35. Cantwell M, Hua T, Pappas J, et al. Acquired CD40-ligand deficiency in chronic lymphocytic leukemia. Nat Med. 1997; 3:984-989. [PubMed: 9288724]

36. Noelle RJ, Roy M, Shepherd DM, et al. A39-kDa protein on activated helper T cells binds CD40 and transduces the signal for cognate activation of B cells. Proc Natl Acad Sci U S A. 1992; 89:6550-6554. [PubMed: 1378631]

37. Ranheim EA, Kipps TJ. Activated T cells induce expression of B7/ BB1 on normal or leukemic B cells through a CD40-dependent signal. J Exp Med. 1993; 177:925-935. [PubMed: 7681471]

38. Dazzi F, D'Andrea E, Biasi G, et al. Failure of B cells of chronic lymphocytic leukemia in presenting soluble and alloantigens. Clin Immunol Immunopathol. 1995; 75:26-32. [PubMed: 7882591]

39. Van den Hove LE, Van Gool SW, Vandenberghe P, et al. CD40 triggering of chronic lymphocytic leukemia B cells results in efficient alloantigen presentation and cytotoxic T lymphocyte induction by up-regulation of CD80 and CD86 costimulatory molecules. Leukemia. 1997; 11:572-580. [PubMed: 9096698]

40. Kato K, Cantwell MJ, Sharma S, et al. Gene transfer of CD40-ligand induces autologous immune recognition of chronic lymphocytic leukemia B cells. J Clin Invest. 1998; 101:1133-1141. [PubMed: 9486984]

41. Wierda WG, Cantwell MJ, Woods SJ, et al. CD40-ligand (CD154) gene therapy for chronic lymphocytic leukemia. Blood. 2000; 96(9):2917-2924. [PubMed: 11049967]

42. Wierda WG, Castro JE, Aguillon R, et al. A phase I study of immune gene therapy for patients with CLL using a membranestable, humanized CD154. Leukemia. 2010; 24:1893-1900. [PubMed: 20882050] This study of immune gene therapy with humanized CD154 demonstrated clinical responses and avoided the induction of an immune response seen with murine CD154.

43. Jak M, van Bochove GG, van Lier RA, et al. CD40 stimulation sensitizes CLL cells to rituximabinduced cell death. Leukemia. 2011; 25:968-978. [PubMed: 21403646] 
44. Scielzo C, Apollonio B, Scarfò L, et al. The functional in vitro response to CD40 ligation reflects a different clinical outcome in patients with chronic lymphocytic leukemia. Leukemia. 2011; 25:1760-1767. [PubMed: 21709686]

45. Chen CI, Bergsagel PL, Paul H, et al. Single-agent lenalidomide in the treatment of previously untreated chronic lymphocytic leukemia. J Clin Oncol. 2010; 29:1175-1181. [PubMed: 21189385] This study shows that single-agent lenalidomide has efficacy comparable to that of cytotoxic agents in treating previously untreated CLL.

46. Badoux XC, Keating MJ, Wen S, et al. Lenalidomide as initial therapy of elderly patients with chronic lymphocytic leukemia. Blood. 2011; 118:3489-3498. [PubMed: 21725050] This trial demonstrates the efficacy of lenalidomide in elderly patients with CLL, highlighting its potential as a treatment for this subgroup.

47. Andritsos LA, Johnson AJ, Lozanski G, et al. Higher doses of lenalidomide are associated with unacceptable toxicity including life-threatening tumor flare in patients with chronic lymphocytic leukemia. J Clin Oncol. 2008; 26:2519-2525. [PubMed: 18427150]

48. Chanan-Khan A, Miller KC, Lawrence D, et al. Tumor flare reaction associated with lenalidomide treatment in patients with chronic lymphocytic leukemia predicts clinical response. Cancer. 2010; 117:2127-2135. [PubMed: 21523725]

49. Brown JR, Abramson J, Hochberg E, et al. A phase I study of lenalidomide in combination with fludarabine and rituximab in previously untreated CLL/SLL. Leukemia. 2010; 24:1972-1975. [PubMed: 20844565]

50. Corral LG, Haslett PA, Muller GW, et al. Differential cytokine modulation and T cell activation by two distinct classes of thalidomide analogues that are potent inhibitors of TNF-alpha. J Immunol. 1999; 163:380-386. [PubMed: 10384139]

51. LeBlanc R, Hideshima T, Catley LP, et al. Immunomodulatory drug costimulates $\mathrm{T}$ cells via the B7-CD28 pathway. Blood. 2004; 103:1787-1790. [PubMed: 14512311]

52. Haslett PA, Hanekom WA, Muller G, et al. Thalidomide and a thalidomide analogue drug costimulate virus-specific CD8+ T cells in vitro. J Infect Dis. 2003; 187:946-955. [PubMed: 12660941]

53. Lapalombella R, Andritsos L, Liu Q, et al. Lenalidomide treatment promotes CD154 expression on CLL cells and enhances production of antibodies by normal B Cells through a PI3-kinase dependent pathway. Blood. 2009; 115:2619-2629. [PubMed: 19965642]

54. Galustian C, Meyer B, Labarthe MC, et al. The anti-cancer agents lenalidomide and pomalidomide inhibit the proliferation and function of T regulatory cells. Cancer Immunol Immunother. 2009; 58:1033-1045. [PubMed: 19009291]

55. Davies FE, Raje N, Hideshima T, et al. Thalidomide and immunomodulatory derivatives augment natural killer cell cytotoxicity in multiple myeloma. Blood. 2001; 98:210-216. [PubMed: 11418482]

56. Idler I, Giannopoulos K, Zenz T, et al. Lenalidomide treatment of chronic lymphocytic leukaemia patients reduces regulatory T cells and induces Th17 T helper cells. Br J Haematol. 2009; 148:948-950. [PubMed: 19961483]

57. Gribben JG, Hosing C, Maloney DG. Stem cell transplantation for indolent lymphoma and chronic lymphocytic leukemia. Biol Blood Marrow Transplant. 2011; 17:S63-S70. [PubMed: 21195313]

58. Rosenberg SA, Yang JC, Sherry RM, et al. Durable complete responses in heavily pretreated patients with metastatic melanoma using T-cell transfer immunotherapy. Clin Cancer Res. 2011; 17:4550-4557. [PubMed: 21498393]

59. Rooney CM, Smith CA, Ng CY, et al. Infusion of cytotoxic T cells for the prevention and treatment of Epstein-Barr virus-induced lymphoma in allogeneic transplant recipients. Blood. 1998; 92:1549-1555. [PubMed: 9716582]

60. Morgan RA, Dudley ME, Wunderlich JR, et al. Cancer regression in patients after transfer of genetically engineered lymphocytes. Science. 2006; 314:126-129. [PubMed: 16946036]

61. June CH, Blazar BR, Riley JL. Engineering lymphocyte subsets: tools, trials and tribulations. Nat Rev Immunol. 2009; 9:704-716. [PubMed: 19859065]

62. Cartellieri M, Bachmann M, Feldmann A, et al. Chimeric antigen receptor-engineered T cells for immunotherapy of cancer. J Biomed Biotechnol. 2010; 2010:956304. [PubMed: 20467460] 
63. Koehler P, Schmidt P, Hombach AA, et al. Engineered T cells for the adoptive therapy of B-cell chronic lymphocytic leukaemia. Adv Hematol. 2011; 2012:595060. [PubMed: 21837241]

64. Hudecek M, Schmitt TM, Baskar S, et al. The B-cell tumor associated antigen ROR1 can be targeted with T-cells modified to express a ROR1-specific chimeric antigen receptor. Blood. 2010; 116:4532-4541. [PubMed: 20702778]

65. Giordano Attianese GM, Marin V, Hoyos V, et al. In vitro and in vivo model of a novel immunotherapy approach for chronic lymphocytic leukemia by anti-CD23 chimeric antigen receptor. Blood. 2011; 117:4736-4745. [PubMed: 21406718]

66. Kochenderfer JN, Yu Z, Frasheri D, et al. Adoptive transfer of syngeneic T cells transduced with a chimeric antigen receptor that recognizes murine CD19 can eradicate lymphoma and normal B cells. Blood. 2010; 116:3875-3886. [PubMed: 20631379]

67. Brentjens RJ, Latouche JB, Santos E, et al. Eradication of systemic B-cell tumors by genetically targeted human T lymphocytes costimulated by CD80 and interleukin-15. Nat Med. 2003; 9:279286. [PubMed: 12579196]

68. Maher J, Brentjens RJ, Gunset G, et al. Human T-lymphocyte cytotoxicity and proliferation directed by a single chimeric TCRzeta /CD28 receptor. Nat Biotechnol. 2002; 20:70-75. [PubMed: 11753365]

69. Kochenderfer JN, Wilson WH, Janik JE, et al. Eradication of Blineage cells and regression of lymphoma in a patient treated with autologous T cells genetically-engineered to recognize CD19. Blood. 2010; 116:4099-4102. [PubMed: 20668228]

70. Kochenderfer JN, Dudley ME, Stetler-Stevenson M, et al. A Phase I Clinical Trial of Treatment of B-Cell Malignancies with Autologous Anti-CD19-CAR-Transduced T cells. Blood. 2010; 116 Abstract 2865 .

71. Brentjens R, Yeh R, Bernal Y, et al. Treatment of chronic lymphocytic leukemia with genetically targeted autologous $\mathrm{T}$ cells: case report of an unforeseen adverse event in a phase I clinical trial. Mol Ther. 2010; 18:666-668. [PubMed: 20357779]

72. Brentjens RJ, Riviere I, Park JH, et al. Safety and persistence of adoptively transferred autologous CD19-targeted T cells in patients with relapsed or chemotherapy refractory B-cell leukemias. Blood. 2011

73. Porter DL, Levine BL, Kalos M, et al. Chimeric Antigen Receptor-Modified T cells in Chronic Lymphoid Leukemia. N Engl J Med. 2011; 365:725-733. [PubMed: 21830940] This case report provides a proof-of-concept base for the use of chimeric antigen receptor-modified $\mathrm{T}$ cells in CLL.

74. Kalos M, Levine BL, Porter DL, et al. T cells with chimeric antigen receptors have potent antitumor effects and can establish memory in patients with advanced leukemia. Sci Transl Med. 2011; 3:95ra73.

75. Milone MC, Fish JD, Carpenito C, et al. Chimeric receptors containing CD137 signal transduction domains mediate enhanced survival of T cells and increased antileukemic efficacy in vivo. Mol Ther. 2009; 17:1453-1464. [PubMed: 19384291]

76. Aue G, Njuguna N, Tian X, et al. Lenalidomide-induced upregulation of CD80 on tumor cells correlates with T-cell activation, the rapid onset of a cytokine release syndrome and leukemic cell clearance in chronic lymphocytic leukemia. Haematologica. 2009; 94:1266-1273. [PubMed: 19734418]

77. Urba WJ, Longo DL. Redirecting T cells. N Engl J Med. 2011; 365:754-757. [PubMed: 21830939]

78. Wherry EJ. T cell exhaustion. Nat Immunol. 2011; 12:492-499. [PubMed: 21739672]

79. Boissel L, Betancur M, Wels WS, et al. Transfection with mRNA for CD19 specific chimeric antigen receptor restores NK cell mediated killing of CLL cells. Leuk Res. 2009; 33:1255-1259. [PubMed: 19147228] 


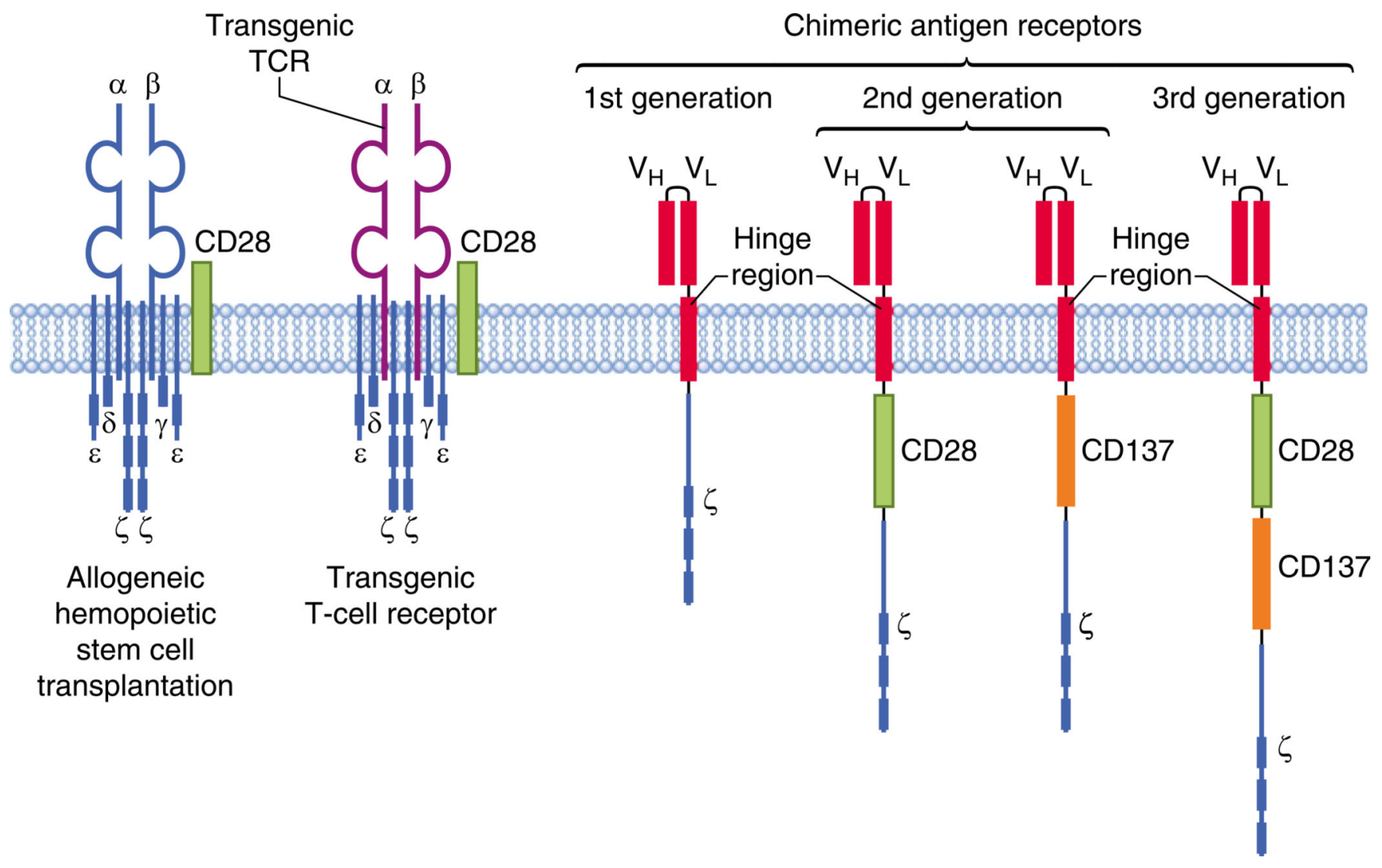

Fig. 1.

Adoptive T-cell therapies in chronic lymphocytic leukemia. T-cell mediated therapies include the adoptive transfer of allogeneic $\mathrm{T}$ cells as part of hemopoietic stem cell transplantation, the transfer of allogeneic or autologous $\mathrm{T}$ cells with a transgenic T-cell receptor, and the transfer of T cells with chimeric antigen receptors (CARs). Early studies used CARTcells without a co-stimulatory domain (1st generation). Current trials are using 2nd-generation CAR T cells, with either a CD28 or CD137 (4-1BB) co-stimulatory domain. Preclinical studies are investigating the combination of co-stimulatory domains ( $3 \mathrm{rd}$ generation) 